https://doi.org/10.15407/ujpe64.8.689

V.M. GORKAVENKO

Taras Shevchenko National University of Kyiv, Ukraine

(64, Volodymyrs'ka Str., Kyiv 01601, Ukraine; e-mail: gorkavol@gmail.com)

\title{
SEARCH FOR HIDDEN PARTICLES IN INTENSITY FRONTIER EXPERIMENT SHiP
}

\begin{abstract}
Despite the undeniable success of the Standard Model of particle physics (SM), there are some phenomena (neutrino oscillations, baryon asymmetry of the Universe, dark matter, etc.) that SM cannot explain. This phenomena indicate that the SM have to be modified. Most likely, there are new particles beyond the SM. There are many experiments to search for new physics that can be can divided into two types: energy and intensity frontiers. In experiments of the first type, one tries to directly produce and detect new heavy particles. In experiments of the second type, one tries to directly produce and detect new light particles that feebly interact with SM particles. The future intensity frontier SHiP experiment (Search for $\boldsymbol{H i d d e n ~} \boldsymbol{P}$ articles) at the CERN SPS is discussed. Its advantages and technical characteristics are given.
\end{abstract}

Ke ywords: physics beyond the Standard Model, hidden particles, hidden sectors, renormalizable portals, intensity frontier experiment, SHiP, SPS.

\section{Introduction}

The Standard Model of particle physics (SM) [1-3] was developed in the mid-1970s. It is one of the greatest successes of physics. It is experimentally tested with high precision for the processes of electroweak and strong interactions with the participation of elementary particles up to the energy scale $\sim 100 \mathrm{GeV}$ and for individual processes up to several TeV. It predicted a number of particles, last of them (Higgs boson) has been observed in 2012. However, the SM cannot explain several phenomena in particle physics, astrophysics, and cosmology. Namely: the SM does not provide the dark matter candidate; the SM does not explain neutrino oscillations and the baryon asymmetry of the Universe; the SM cannot solve the strong CP problem in particle physics, the primordial perturbations problem and the horizon problem in cosmology, etc.

The presence of the problems in the SM indicates the incompleteness of the Standard Model and the existence of as yet "hidden" sectors with particles of a new physics. Although it may seem surprising, but some of the above-mentioned SM problems really can be solved with help either heavy or light new particles. Neutrino oscillations and the smallness of the active neutrino mass can be explained as with

(C) V.M. GORKAVENKO, 2019

ISSN 2071-0194. Ukr. J. Phys. 2019. Vol. 64, No. 8 help of a new particle with sub-eV mass, as well as with help of heavy particles of the GUT scale, see, e.g., [4]. The same can be said about the problem of baryon asymmetry of the Universe and dark matter problem: physics on the very different scales can be responsible for it, see, e.g., [5].

Can the new light particles exist in the SM extensions? The answer is positive. There are many theories beyond SM that have light particles in the spectrum (e.g., GUT, SUSY, theories with extra dimensions), see, e.g., [6]. Light particles in those theories can be, e.g., (pseudo)-Goldstone bosons that were produced as a result of the spontaneous breaking of some not exact symmetry. Alternatively, a particle can be massless at the tree level, but it can obtain a light mass as a result of loops-involving corrections.

So, two answers on the question "why do we not observe particles of the new physics?" are possible. First, the new particles can be very heavy (e.g., with the mass $M_{X} \gtrsim 100 \mathrm{TeV}$ ), so they cannot be directly produced at the present-day powerful accelerators like LHC. On other hand, the new particles can be light (with mass below or of order of the electroweak scale) and can feebly interact with particles of the SM (otherwise, we would have already seen them in the experiments). In this case, the light new particles can be produced at many high-energy experiments, but it were not still observed due to the 

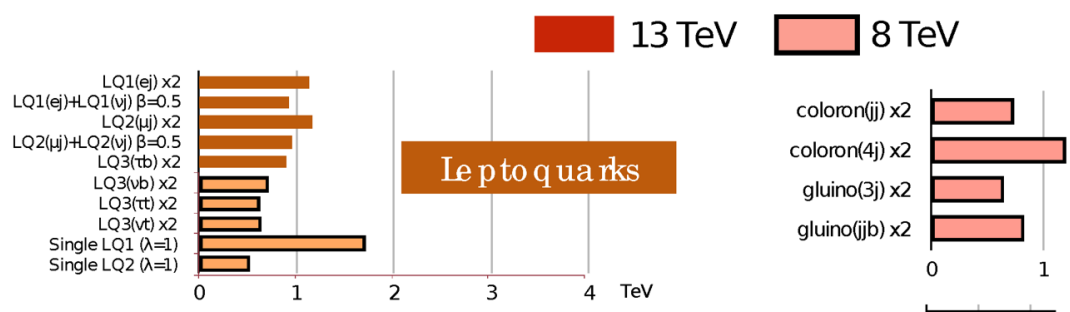

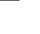

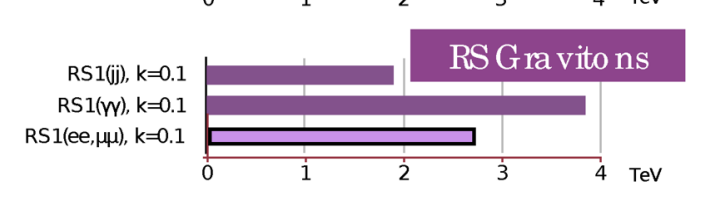

\section{CMS Preliminary}

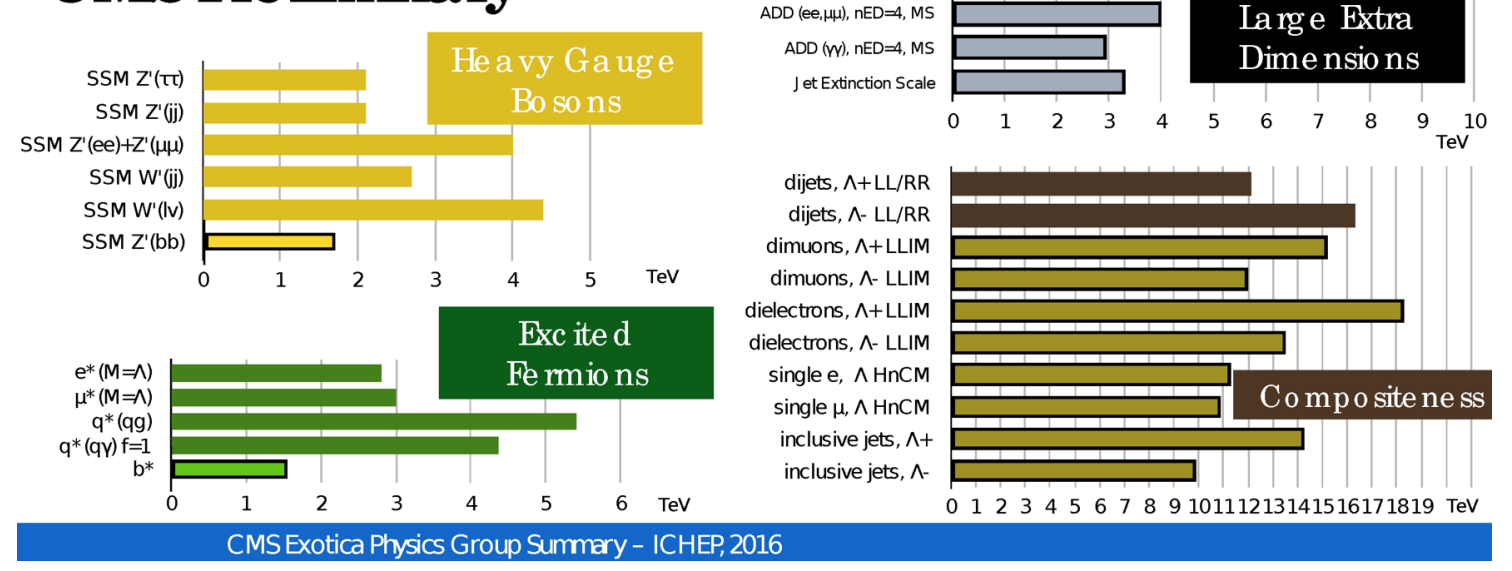

Fig. 1. Search for new particles beyond SM with mass at the TeV region at CERN CMS

extreme rarity of events with their production and to the complexity of their detection.

Based on the above, there are two types of particle search experiments.

First of them is energy frontier experiments like those at LHC or Fermilab. In these experiments, one tries to directly produce and detect new heavy particles assuming that the coupling of new particles to the SM particles is not very small. The new particles with mass of several $\mathrm{TeV}$ are actively searched in such experiments, see Fig. 1. Last decades, a lot of attention were paid to the energy frontier experiments.

Second of them is intensity frontier experiments. In this experiments, we try to search for the particles that feebly interact with the SM particles. So, in the intensity frontier experiments, we search for very rare events. For the successful production of hidden particles (to compensate their feeble interaction), those experiments must have the largest possible luminosity. In this sense, the beam-dump experiments are good as the intensity frontier experiments to seek the GeV-scale hidden particles, because of their luminosities is several orders of magnitude larger than those at colliders. The detection of hidden particles is possible only due to observing their decays into the SM particles. So, these experiments must be backgroundfree. Because of the feeble interaction with the SM particles, one can expect their small decay width and long lifetime (here, we suppose that a hidden particle does not decay in non-SM channels, or the corresponding partial decay width is very small). So, the detector have to be placed as far as possible from the point of the production of hidden particles.

The intensity frontier experiments have been paid much less attention in the recent years. These experiments include PS 191 (early 1980s), CHARM (1980s), NuTeV (1990s), DONUT (late 1990s - early 2000). However, as was shown in [7,9], the search for the new physics in the region of masses below the electroweak scale is not sufficiently investigated. 
The difference between the energy and intensity frontier experiments for seeking the hidden particles can be schematically illustrated with the help of Fig. 2.

In this paper, we consider the future intensity frontier SHiP (Search for Hidden Particles) beam-dump experiment at the CERN Super Proton Synchrotron (SPS) accelerator. Its advantages and technical characteristics will be considered, and the class of theories that can be tested on SHiP will be discussed.

\section{Interaction of New \\ Particles with the SM Particles. Portals}

If we will focused on detecting a new light particle, we have understand that this particle can originate from the large number of beyond-SM theories that predict different parameters for it (masses of new particles and their coupling to the SM particles). In particular, such relatively light particles can be mediators due to the interaction with particles of the SM and very heavy particles of "hidden sectors". Those light particles can be coupled to the Standard Model sectors either via renormalizable interactions with small dimensionless couplings ("portals") or by higher-dimensional operators suppressed by the dimensionful couplings $\Lambda^{-n}$ corresponding to a new energy scale of the hidden sector [7].

Because of a limited number of possible types of particles (scalar, pseudoscalar, vector, pseudovector, fermion), there is limited number of possible effective Lagrangians of interaction of such particles with the SM particles that satisfy the Lorentz conditions and gauge invariance ones.

Renormalizable portals can be classified into the following 3 types:

Vector portal: new particles are vector Abelian fields $\left(A_{\mu}^{\prime}\right)$ with the field strength $F_{\mu \nu}^{\prime}$ that couple to the hypercharge field $F_{Y}^{\mu \nu}$ of the SM as

$\mathcal{L}_{\text {Vectorportal }}=\epsilon F_{\mu \nu}^{\prime} F_{Y}^{\mu \nu}$

where $\epsilon$ is a dimensionless coupling characterising the mixing between a new vector field with the fields of $Z$-bosons and photons.

Scalar portal: new particles are neutral singlet scalars, $S_{i}$, that couple to the Higgs field

$\mathcal{L}_{\text {Scalarportal }}=\left(\lambda_{i} S_{i}+g_{i} S_{i}^{2}\right)\left(H^{\dagger} H\right)$,

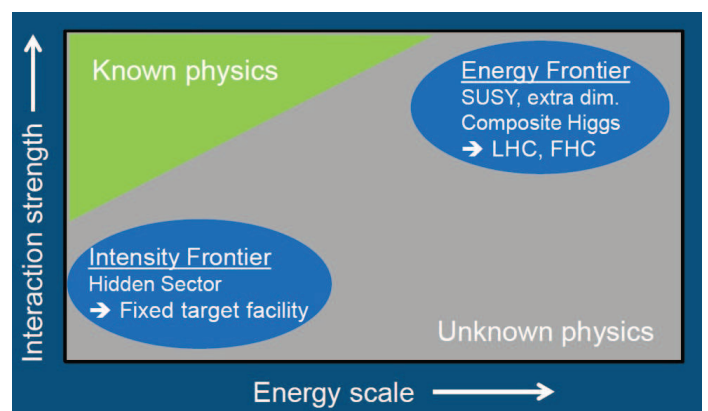

Fig. 2. Different strategies for the search for hidden particles in the energy and intensity frontier experiments

where $\lambda_{i}$ are dimensionless couplings, and $g_{i}$ are the couplings with a dimension of mass.

Neutrino portal: new particles are neutral singlet fermions $N_{I}$

$\mathcal{L}_{\text {Neutrinoportal }}=F_{\alpha I} \bar{L}_{\alpha} \tilde{H} N_{I}$,

where index $\alpha=e, \mu, \tau$ corresponds to the lepton flavors, $L_{\alpha}$ is for the lepton doublet, $F_{\alpha I}$ is for the new matrix of the Yukawa constants, and $\tilde{H}=i \sigma_{2} H^{*}$.

Non-renormalizable couplings of new particles to the SM operators are also possible. For example, pseudo-scalar axion-like particles $A$ couple to SM as

$$
\begin{aligned}
& \mathcal{L}_{\mathrm{A}}=\sum_{f} \frac{C_{A f}}{2 f_{a}} \bar{f} \gamma^{\mu} \gamma^{5} f \partial_{\mu} A- \\
& -\frac{\alpha}{8 \pi} \frac{C_{A \gamma}}{f_{a}} F_{\mu \nu} \tilde{F}^{\mu \nu} A-\frac{\alpha_{3}}{8 \pi} \frac{C_{A 3}}{f_{a}} G_{\mu \nu}^{b} \tilde{G}^{b \mu \nu} A,
\end{aligned}
$$

where $f=\{$ quarks, leptons, neutrinos $\}, F_{\mu \nu}$ is the electromagnetic field strength tensor, $G_{\mu \nu}^{b}$ the field strength for a strong force, and the dual field strength tensors are defined as $\tilde{Q}^{\mu \nu}=\frac{1}{2} \epsilon^{\mu \nu \rho \sigma} Q_{\rho \sigma}$.

Another important example is a Chern-Simons-like gauge interaction [8] of a new pseudo-vector $X_{\mu}$ particle

$\mathcal{L}_{1}=\frac{C_{Y}}{\Lambda_{Y}^{2}} \cdot X_{\mu}\left(\mathfrak{D}_{\nu} H\right)^{\dagger} H B_{\lambda \rho} \cdot \epsilon^{\mu \nu \lambda \rho}+$ h.c.
$\mathcal{L}_{2}=\frac{C_{S U(2)}}{\Lambda_{S U(2)}^{2}} \cdot X_{\mu}\left(\mathfrak{D}_{\nu} H\right)^{\dagger} F_{\lambda \rho} H \cdot \epsilon^{\mu \nu \lambda \rho}+$ h.c.,

where the $\Lambda_{Y}, \Lambda_{S U(2)}$ are new scales of the theory, $C_{Y}, C_{S U(2)}$ are new dimensionless coupling constants, and $B_{\mu \nu}, F_{\mu \nu}$ are the field strength tensors of the 


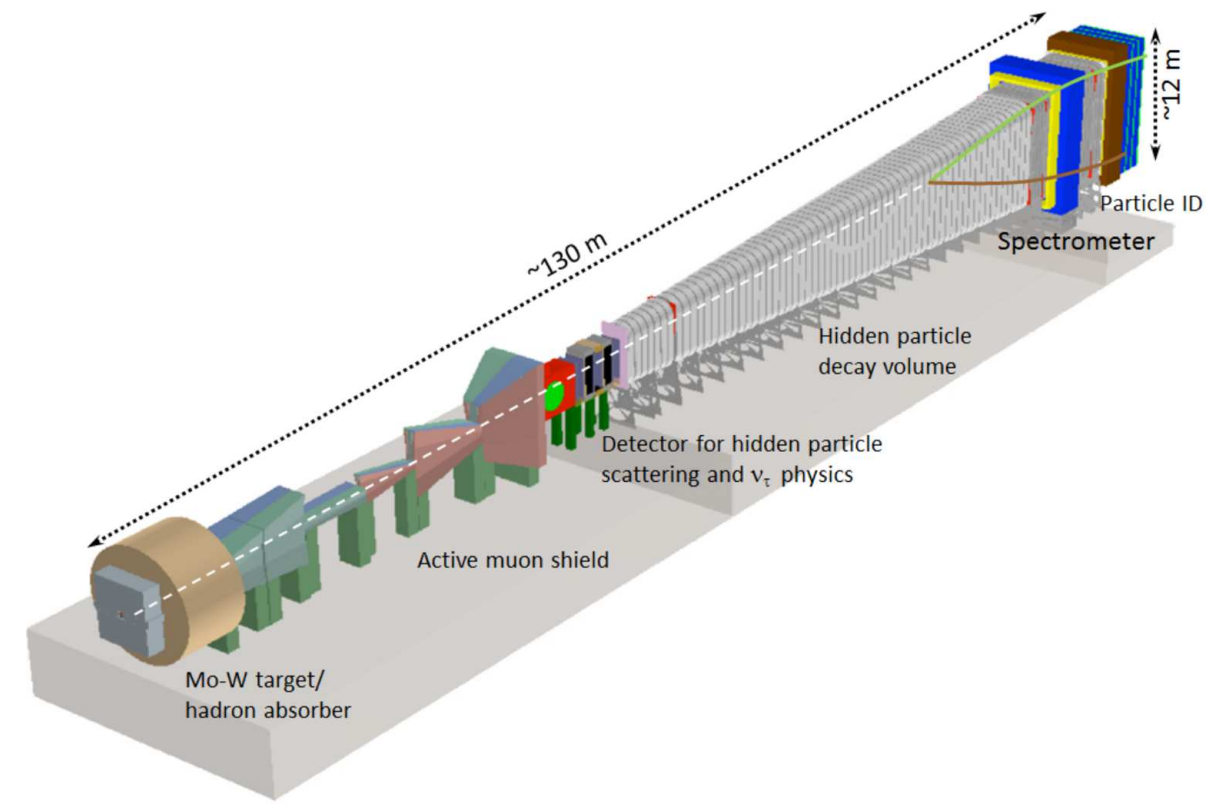

Fig. 3. General scheme of the SHiP facility

$U_{Y}(1)$ and $S U_{W}(2)$ gauge fields. After the spontaneous symmetry breaking of the Higgs field, this interaction is effectively reduced to a renormalizable interaction of the form

$\mathcal{L}_{\mathrm{CS}}=c_{z} \epsilon^{\mu \nu \lambda \rho} X_{\mu} Z_{\nu} \partial_{\lambda} Z_{\rho}+c_{\gamma} \epsilon^{\mu \nu \lambda \rho} X_{\mu} Z_{\nu} \partial_{\lambda} A_{\rho}+$

$+c_{w} \epsilon^{\mu \nu \lambda \rho} X_{\mu} W_{\nu}^{-} \partial_{\lambda} W_{\rho}^{+}$.

So, from the experimental point of view, one has to test all of the above-mentioned possible new interactions in the wide range of new particle masses and couplings.

\section{SHiP Experiment}

The SHiP experiment was first proposed in 2013 [10]. The technical proposal was presented in 2015 [11]. The theoretical background, main channels of production and decay of new particles, and preliminary estimations of the sensitivity region for different portals for the SHiP experiment were considered in 2016 [7]. Somewhat later, the clarifying complementary works were published [12-15]. Currently, the SHiP collaboration [16] includes nearly 250 scientists from 53 institutions. The experiment will begin its work allegedly in 2026 year [17].

The main goal of the future SHiP beam-dump experiment at the CERN SPS accelerator is to search for the new physics in the region of feebly interacting long-lived light particles including Heavy Neutral Leptons (HNL), vector, scalar, axion portals to the Hidden Sector, and light supersymmetric particles. The experiment provides great opportunities for the study of neutrino physics as well.

Now, we describe the work of the SHiP experiment, see Fig. 3. A beam line from the CERN SPS accelerator will transmit $400-\mathrm{GeV}$ protons at the SHiP. The proton beam will strike in a Molybdenum and Tungsten fixed target at a center-of-mass energy $E_{\mathrm{CM}} \approx 27 \mathrm{GeV}$. Approximately $2 \times 10^{20}$ protontarget collisions are expected in 5 years of the SHiP operation. The great number of the SM particles and hadrons will be produced under such collisions. Hidden particles are expected to be predominantly produced in the decays of the hadrons.

The main concept of the SHiP functioning is following. Almost all the produced SM particles should be either absorbed or deflected in a magnetic field (muons). Remaining events with SM particles can be rejected using specially developed cuts. If the hidden particles will decay into SM particles inside the decay volume, the last will be detected. This will mean the existence of hidden particles.

So, the target will be followed by a 5 -m-long iron hadron absorber. It will absorb the hadrons and the 
electromagnetic radiation from the target, but the decays of mesons result in a large flux of muons and neutrinos. After the hadron stopper, a system of shielding magnets (which extends over a length of $\sim 40 \mathrm{~m}$ ) is located to deflect muons away from the fiducial decay volume [12].

Despite the aim to search for the long-lived particles, the sensitive volume should be situated as close as possible to the proton target due to relatively large transverse momenta of the hidden particles with respect to the beam axis. The minimum distance is determined by the necessity for the system to absorb the electromagnetic radiation and hadrons produced in the proton-target collisions and to reduce the beaminduced muon flux.

The system of detectors of the SHiP consists of two parts. Just after the hadron absorber and muon shield, the detector system for recoil signatures of hidden-sector particle scattering and for neutrino physics is located. The neutrino detector has mass of nearly 10 tons. The study of neutrino physics is based on a hybrid detector similar to the detector of the OPERA Collaboration [18]. In addition, this system allows one to detect and veto charged particles produced outside the main decay volume.

The second detector system consist of the fiducial decay volume that is contained in a nearly 50 -m-long rectangular vacuum tank. In order to suppress the background from neutrinos interacting in the fiducial volume, it is maintained at a pressure of $O\left(10^{-3}\right)$ bar. The decay volume is surrounded by background taggers to tag the neutrino and muon inelastic scatterings in the surrounding structures, which may produce long-lived neutral Standard Model particles

Modification of the SM that can be tested on SHiP depending on final states of the hidden particles decay

\begin{tabular}{|c|c|c|}
\hline Decay modes & Final states & Models tested \\
\hline Meson and lepton & $\begin{array}{l}\pi l, K l, l \\
(l=e, \mu, \tau)\end{array}$ & $\begin{array}{l}\nu \text { portal, HNL, } \\
\text { SUSY neutralino }\end{array}$ \\
\hline Two leptons & $e^{+} e^{-}, \mu^{+} \mu^{-}$ & $\begin{array}{l}\mathrm{V}, \mathrm{S} \text { and A portals, } \\
\text { SUSY s-goldstino }\end{array}$ \\
\hline Two mesons & $\pi^{+} \pi^{-}, K^{+} K^{-}$ & $\begin{array}{l}\text { V, S and A portals, } \\
\text { SUSY s-goldstino }\end{array}$ \\
\hline 3 bodies & $l^{+} l^{-} \nu$ & HNL, neutralino \\
\hline
\end{tabular}

ISSN 2071-0194. Ukr. J. Phys. 2019. Vol. 64, No. 8 whose decay can mimic signal events. The vacuum tank is followed by a large spectrometer with a rectangular acceptance of $5 \mathrm{~m}$ in width and $10 \mathrm{~m}$ in height and a calorimeter. The system is constructed in such a way to detect as many final states as possible in order to be sensitive to a very wide range of models that can be tested. With the help of Table, one can see what modification of the SM is tested depending on final states of the hidden particles decay.

It should be noted that the SHiP experiment gives great opportunities for the study of neutrino physics. As a result of nearly $2 \times 10^{20}$ proton-target collisions, $N_{\nu_{\tau}}=5.7 \times 10^{15} \nu_{\tau}$ and $\nu_{\bar{\tau}}$ neutrino, $N_{\nu_{e}}=$ $=5.7 \times 10^{18}$ electron neutrino, and $N_{\nu_{\mu}}=3.7 \times 10^{17}$ muon neutrino will be produced. It is expected to detect nearly $10^{4} \tau$-neutrino and at first to detect anti $\tau$-neutrino. It is very important, because only 14 $\tau$-neutrino candidates by the experiment DONUT in Fermilab and $10 \tau$-neutrino candidates by the experiment OPERA in CERN were found till now. No event with anti $\tau$-neutrino was still observed.

\section{Conclusions}

There are some indisputable phenomena that point to the fact that SM has to be modified and complemented by a new particle (particles). We are sure that there is a new physics, but we do not know where to search for it. There are many theoretical possibilities to modify the SM by scalar, pseudoscalar, vector, pseudovector, or fermion particles of the new physics. These particles may be sufficiently heavy on the electroweak scale and the scale of energy of the present colliders. But these particles may be light (with masses less than that on the electroweak scale) and may feebly interact with the SM particles. The main task now is to experimentally observe particles of the new physics.

Since the possibilities for increasing the energies of the present colliders are limited by high costs, and the heavy new particles are difficult to be produced, it seems reasonable to check another variant and to find light particles of the new physics in intensity frontier experiments.

The goal of the SHiP experiment is to search for the new physics in the region of feebly interacting longlived light particles including HNL, vector, scalar, axion particles with mass $\leq 10 \mathrm{GeV}$. There are theoretical predictions for the sensitivity region of the SHiP 
experiment for each type of new-physics particles (in the mass versus coupling constant coordinates). The experiment will provide great opportunities for the study of neutrino physics as well.

Since the idea of searching for new light feebly interacting particles is very tempting and promising, there are another projects such as REDTOP at the PS beam lines, NA64 ++ , NA62 ++ , LDMX, AWAKE, KLEVER at the SPS beam lines, and FASER, MATHUSLA, CODEX-b at the LHC. All these experiments are compared and summarized in [17]. It is possible that great discoveries in particle physics are right ahead.

The work was presented on the conference "New trends in high-energy physics", May 12-18, Odessa, Ukraine. I also thank Kyrylo Bondarenko for the useful discussion and helpful comments.

1. S.L. Glashow. Partial symmetries of weak interactions. Nucl. Phys. 22, 579 (1961).

2. S. Weinberg. A Model of leptons. Phys. Rev. Lett. 19, 1264 (1967).

3. A. Salam. Weak and electromagnetic interactions. In: Proc. of 8th Nobel Symposium. Edit. by N. Svartholm (Almquist and Wiksells, 1968), p. 367.

4. A. Strumia, F. Vissani. Neutrino masses and mixings and... arXiv:hep-ph/0606054 (2010).

5. D.S. Gorbunov, V.A. Rubakov. Introduction to the Theory of the Early Universe: Hot Big Bang Theory (World Scientific, 2011).

6. W. de Boer. Grand unified theories and supersymmetry in particle physics and cosmology. Prog. Part. Nucl. Phys. 33, 201 (1994).

7. S. Alekhin. et al. A facility to search for hidden particles at the CERN SPS: the SHiP physics case. Rept. Prog. Phys. 79, 124201 (2016).

8. I. Antoniadis, A. Boyarsky, S. Espahbodi, O. Ruchayskiy, J.D. Wells. Anomaly driven signatures of new invisible physics at the Large Hadron Collider. Nucl. Phys. B 824, 296 (2010).

9. J. Alexander et al. Dark Sectors 2016 Workshop: Community report. arXiv:1608.08632, FERMILAB-CONF-16-421 (2016).

10. W. Bonivento et al. Proposal to search for Heavy Neutral Leptons at the SPS. arXiv:1310.1762 (2013).
11. M. Anelli et al. A facility to Search for Hidden Particles (SHiP) at the CERN SPS. arXiv:1504.04956, CERNSPSC-2015-016, SPSC-P-350 (2015).

12. A. Akmete et al. The active muon shield in the SHiP experiment. JINST 12, P05011 (2017).

13. C. Ahdida et al. Sensitivity of the SHiP experiment to Heavy Neutral Leptons. J. High Energ. Phys. 2019, 77 (2019).

14. C. Ahdida et al. The experimental facility for the Search for Hidden Particles at the CERN SPS. JINST 14, P03025 (2019).

15. I. Boiarska, K. Bondarenko, A. Boyarsky, V. Gorkavenko, M. Ovchynnikov, A. Sokolenko. Phenomenology of GeVscale scalar portal. arXiv:1904.10447v2 (2019).

16. https://ship.web.cern.ch/ship.

17. J. Beacham et al. Physics Beyond Colliders at CERN: Beyond the Standard Model Working Group Report. arXiv:1901.09966, CERN-PBC-REPORT-2018-007 (2019).

18. R. Acquafredda et al. OPERA collaboration. The OPERA experiment in the CERN to Gran Sasso neutrino beam. JINST 4, P04018 (2009).

Received 08.07.19

В.М. Горкавенко

ПОШУК ЧАСТИНОК НОВОї ФІЗИКИ В ЕКСПЕРИМЕНТІ SHiP

Р е $з$ ю м е

Незважаючи на величезні успіхи Стандартної Моделі фізики елементарних частинок (CM), існують окремі явища (нейтринні осциляції, баріонна асиметрія Всесвіту, темна матерія тощо), які CM пояснити не в змозі. Дані явища вказують на необхідність модифікації СМ та введення нових частинок. Експерименти з пошуку частинок нової фізики можна розділити на два типи: експерименти, в яких намагаються досягти найбільшої енергї частинок, що зіштовхуються, та експерименти, в яких намагаються досягти найбільшої кількості необхідних реакцій. В експериментах першого типу намагаються безпосередньо утворити та зареєструвати нові важкі частинки. В експериментах другого типу намагаються безпосередньо утворити та зареєструвати нові легкі частинки, що слабко взаємодіють з частинками СМ. В роботі обговорюється майбутній експеримент 3 високою інтенсивністю подій SHiP, що проводитиметься на прискорювачі SPS CERN, його технічні характеристики та переваги. 